\title{
RESULTS OF LIQUID BIOPSY STUDIES BY NEXT GENERATION SEQUENCING IN PATIENTS WITH ADVANCED STAGE NON-SMALL CELL LUNG CANCER: SINGLE CENTER EXPERIENCE FROM TURKEY
}

\author{
Buyuksimsek $\mathrm{M}^{1, *}$, Togun $\mathrm{M}^{2}$, Oguz Kara $\mathrm{I}^{1}$, Bisgin $\mathrm{A}^{3,4}$, Boga $\mathrm{I}^{4}$, Tohumcuoglu $\mathrm{M}^{1}$, \\ Ogul A ${ }^{1}$, Evren Yetisir A ${ }^{1}$, Sahin B ${ }^{1}$, Sumbul HE , Mirili C $^{6}$ \\ *Corresponding Author: Mahmut Buyuksimsek, M.D., Department of Oncology, Çukurova University \\ Faculty of Medicine, Sarican, Adana, Turkey. Tel: +90-536-862-20-26. Fax: +90-322-338-70-72. \\ E-mail: mahmutbuyuksimsek@gmail.com
}

\begin{abstract}
Several studies demonstrated the utility of plasmabased cell-free circulating tumor DNA(ccfDNA) in determination of mutations in non-small cell lung cancer (NSCLC). We aimed to report our results of next generation sequencing (NGS) using liquid biopsy in patients with NSCLC. Patients with advanced stage NSCLC were enrolled and their genomic profiling results were recorded. Next generation sequencing targeted panel includes 19 hot-spot genes. The plasma was separated from the peripheral blood sample and ccfDNAs were isolated for NGS. We performed genomic profiling in 100 patients (20 females and 80 males) with a median age of 59.3 (range 26-79). A second liquid biopsy was performed in eight patients who developed progressive disease after the first treatment. The study population had adenocarcinoma (AC) $(n=73)$, squamous cell carcinoma (SCC) $(n=14)$, or NSCLC-NOS (not otherwise specified) $(n=13)$. In the SCC group, three of 14 patients had variants on EGFR and MET genes. In the AC and NSCLC-NOS groups, 39 out of 86 patients $(45.3 \%)$ had variants. The most common one was in the EGFR gene ( $n=27,31.4 \%)$ including seven mutations related to drug resistance and two were polymorphisms. Three patients had both driver

\footnotetext{
${ }^{1}$ Department of Medical Oncology, Çukurova University Faculty of Medicine, Balcali Clinics and Hospital, Adana, Turkey Medicine, Balcali Clinics and Hospital, Adana, Turkey

${ }^{3}$ Medical Genetics Department of Balcali Hospital and Clinics and University Faculty of Medicine, Adana, Turkey and Treatment Center), Adana, Turkey

${ }^{5}$ Department of Internal Medicine, University of Health Sciences, Adana Health Practice and Research Center, Adana, Turkey

${ }^{6}$ Department of Medical Oncology Ataturk University Faculty of Medicine, Erzurum, Turkey
}

${ }^{2}$ Department of Internal Medicine, Çukurova University Faculty of

${ }^{4}$ Çukurova University AGENTEM (Adana Genetic Diseases Diagnosis
\end{abstract}

and resistance mutations ( $E G F R$ T790M, $n=2 ; K R A S$ exon 2 G12S and MET exon 14 E1012K, $n=1)$. Fifteen patients (17.4\%) had an activating EGFR mutation and eight patients $(9.3 \%)$ had variants in the $K R A S$ gene. We reported our results regarding genomic profiling related to treatment using liquid biopsy in patients with NSCLC. Advantages of this method are the non invasiveness and reproducibility.

Keywords: Liquid biopsy; New generation sequencing (NGS); Non-small cell lung cancer (NSCLC).

\section{INTRODUCTION}

Non-small cell lung cancer (NSCLC) is an important cause of morbidity and mortality worldwide. Many patients with NSCLS are diagnosed at advanced stages and treated with targeted therapy and immunotherapy in addition to systemic chemotherapy [1]. The College of American Pathologists (CAP), International Association for the Study of Lung Cancer (IASLC) and Association for Molecular Pathology (AMP) recommended that EGFR, ALK, and ROS1 were necessary tests in advanced stage NSCLC patients whose tumors contain an element of adenocarcinoma (AC) in their 2018 updated testing guideline. Moreover, the results of the recent clinical data indicate those panels including $B R A F, M E T, R E T, E R B B 2$ and $K R A S$, should be used at a minimum [2]. Cell-free circulating tumor DNA (ccfDNA), which is released from the tumor into the systemic circulation, is used in the liquid biopsy [3].

Thompson et al. [4] and Schwaederlé et al. [5] demonstrated that next generation sequencing (NGS) of plasmabased ccfDNA can be used to assess mutations in NSCLC. Several recent retrospective and prospective studies also used plasma samples to decide targeted treatments $[6,7]$. The NGS used for the analysis of ccfDNA involves reading of the DNA strand 10,000-times using deep sequencing and allows determination of the type and frequency of a 
given mutation by bioinformatic analyses. It is possible to identify single base mutations, short insertions and deletions, wide genomic deletions, or rearrangements such as inversion and translocation (by amplifications) using NGS [8]. In the present study, we aimed to present our NGS results of liquid biopsy samples that is increasingly used in clinical practice for NSCLC that comprises several genetic alterations guiding therapeutic opportunities.

\section{MATERIALS AND METHODS}

Patient Selection. A prospective review of $100 \mathrm{pa}-$ tients in Adana, Turkey with a diagnosis of advanced/ metastatic NSCLC, whose physician requested clinical ccfDNA based genomic profiling from January 2017 to January 2019, were performed. All patients included in the analysis had metastatic or inoperable disease. A second liquid biopsy was performed in eight patients who developed progressive disease after the first treatment. All patients provided their written consent to the genomic profiling.

Targeted Multi-Gene Panel Testing. Customized targeted multiple gene panel consisting of 19 genes (AKT1, ALK, BRAF, DDR, ERBB2, ESR1, KIT, KRAS, MAP2K1, NRAS, NTRK, PDGFRA, PIK3CA, PTEN, ROS1, RICTOR, EGFR, MET, FGFRl gene mutations, and RICTOR, EGFR, $M E T, F G F R 1, E R B B 2$ gene amplifications) was used for NGS. The ccfDNA was extracted from whole peripheral blood collected in $10 \mathrm{~mL}$ PaxGene (PreAnalytiX GmbH, Hombrechtikon, Switzerland) biological sample tubes. The results of NGS were obtained after the following steps: separation of plasma from the samples, isolation of ccfDNA from the plasma, target region enrichment in an appropriate quality and quantity, library preparation, clonal amplification and NGS steps. Afterwards, bioinformatic analyses were performed to determine the quality and variant analysis according to the clinical information of the patients to interpret the variants. All the workflow was carried out at Çukurova University AGEN TEM (Adana Genetic Diseases Diagnosis and Treatment Center), Adana, Turkey via the GeneReader NGS system (Qiagen GmbH, Hilden, Germany).

The isolation of ccfDNA was performed using the circulating cfDNA isolation Kit (QIAamp circulating nucleic acid kit; Qiagen $\mathrm{GmbH}$ ) with the help of a vacuum system (QIAvac 24 Plus; Qiagen $\mathrm{GmbH}$ ). The ccfDNA concentrations were determined using fluorometric DNA quantitation device (Qubit 3.0, Thermo Fisher Scientific, Waltham, MA, USA). The samples with adequate DNA concentrations were used for further laboratory workflow. The target enrichment of the region of interest was amplified by PCR (polymerase chain reaction). The bar coding and library preparation step was then performed. Thereafter, the samples were loaded into flowcells to be sequenced in the GeneReader NGS system (Qiagen GmbH).
Bioinformatic Analysis and Interpretation. The most complicated and difficult step is the accurate analysis of the large data from the sequenced NGS samples by an experienced medical geneticist and the team. The data with appropriate quality were selected before the data analysis. The selected data were compared with the reference genome data. The Human Genome Mutation Database (HGMD) (http://www.hgmd.cf.ac.uk/ac/index.php0, Catalogue of Somatic Mutations in Cancer (COSMIC) (https:// cancer. sanger.ac.uk/cosmic), 1000 Genome Frequency and Ingenuity Knowledge Base databases (https://www. internationalgenome.org/1000-genomes-browsers), and SIFT, BSIFT (https://sift.bii.a-star.edu.sg/), PolyPhen-2 (http:// genetics.bwh.harvard.edu/pph2/dbsearch.shtml) and Clin Var (https://www.ncbi.nlm.nih.gov/clinvar/) in silico analysis were used for variant analysis. Then, selected variants were analyzed using bioinformatics tools to classify and evaluate them according to their clinical impacts, for potential influence on the treatment strategies, and to confirm the clinical diagnosis. The low quality variants were also assessed for the samples' status and clinical status of the patient. All the variants were evaluated in two steps: the first one was for its quality and possible effects independent of clinical diagnosis and mostly on the basis of quality control parameters such as for ward/reverse read balance and coverage. The second evaluation was performed for clinical diagnosis and possible effect on the sensitivity and/or the resistance to the treatment protocols.

\section{RESULTS}

A total of 100 patients (20 females and 80 males) with NSCLC who were admitted to our clinic underwent targeted NGS from the liquid biopsy samples. All of the patients were of Turkish ethnic origin. The median age of the patients was 59.3 (range 26-79). While eight of the patients had a repeated genetic testing after treatment, other patients had only one test result. The histological diagnoses were made by the pathologists of our clinic. Seventy-three patients had AC, 14 had squamous cell carcinoma (SCC), and 13 had NSCLC-NOS (not otherwise specified). The study patients were grouped according to their histological lung cancer (LC) types as classified by the National Comprehensive Cancer Network (NCCN) in their targeted treatment recommendations. The demographic data and histologic LC types of the patients are listed in Table 1.

The Potential Targetable Variants in the SCC Group. Three of the 14 patients had variants: one patient had EGFR gene exon 21 driver and exon 20 resistance mutations together and the other two patients had a mutation in the MET gene exon 14 (Table 2).

The Potential Targetable Variants in the AC And NSCLC-NOS Groups. Thirty-nine of the 86 patients 
Table 1. General demographics and histology.

\begin{tabular}{|l|l|l|l|}
\hline $\begin{array}{c}\text { General } \\
\text { Demographics }\end{array}$ & $\boldsymbol{n}$ & \multicolumn{1}{|c|}{ Histology } & $\boldsymbol{n}$ \\
\hline Patients & 100 & SCC & 14 \\
\hline Median age (range) & $59.3(26-79)$ & AC & 73 \\
\hline Males/Females & $80 / 20$ & NSCLC-NOS & 13 \\
\hline
\end{tabular}

SCC: squamous cell carcinoma; $\mathrm{AC}$ : adenocarcinoma;

NSCLC-NOS: non-small cell lung cancer-not otherwise specified.

Table 2. Targetable mutations in squamous cell carcinoma patients.

\begin{tabular}{|c|l|c|}
\hline $\boldsymbol{n}$ & \multicolumn{1}{|c|}{ Activating Mutation(s) } & \multicolumn{1}{c|}{ Resistance Mutation } \\
\hline 24 & $M E T$ exon 14 I491T & \\
\hline 29 & $M E T$ exon 14 V1014V & \\
\hline 56 & $E G F R$ exon 21 L858R & EGFR exon 20 T790M \\
\hline
\end{tabular}

(45.3\%) had at least one variant of the targeted multi-gene panel. Twenty-seven (31.4\%) of the 86 patients had a variant in the EGFR gene, while seven had a resistance related mutation, two had a polymorphism, and three had both driver and resistance related variants (EGFR T790M, $n=$ 2; KRAS exon $2 \mathrm{G} 12 \mathrm{~S}$ and $M E T$ exon $14 \mathrm{E} 1012 \mathrm{~K}, n=1)$.

Fifteen patients (17.4\%) had an activating EGFR mutation (Table 3). Eight patients $(9.3 \%)$ had a KRAS gene variant. One patient had an isolated $A L K$ mutation and another one had an ERBB2 and EGFR gene variant in addition to an $A L K$ mutation. While one patient had an isolated $M E T$ amplification, two patients had a concomitant $E G F R$ resistance related variant. While two patients had an isolated ERBB2 gene variant, another two had EGFR variants and $A L K$ mutation in addition to the $E R B B 2$ variant. One patient had variants in both PDGFRA and KIT genes. Another one had an isolated $B R A F$ variant (Table 4). In the patients who experienced progressive disease after treatment, eight of them underwent a second liquid biopsy sampling after a median duration of 6 months (range 3-12) following the first one. In the second term of sequencing, a new onset $E G F R$ T790M mutation, elimination of the target mutation, and a new onset of $B R A F$ variant were detected. The characteristics and treatment protocols of these eight patients are summarized in Table 5.

Table 3. Detailed Molecular Findings of Adenocarcinoma and NSLC-NOS patients with detectable EGFR mutations

\begin{tabular}{|c|c|c|c|}
\hline Patient Number & Activating Mutation(s) & Resistance Mutation(s) & Polymorphism \\
\hline 2 & \begin{tabular}{|l|} 
EGFR Exon 19 A750P, \\
EGFR Exon 19 L747 E749 del \\
\end{tabular} & & \\
\hline 4 & EGFR Exon 19 & & \\
\hline 6 & EGFR Exon 21 L858R & EGFR Exon 20 T790M & \\
\hline 7 & $\begin{array}{l}\text { EGFR Exon } 20 \text { C797S } \\
\text { EGFR Exon } 21 \text { L858R }\end{array}$ & EGFR Exon 20 T790M & \\
\hline 10 & & EGFR Exon 18 G721V & \\
\hline 12 & $\begin{array}{l}\text { EGFR Exon } 20 \mathrm{~S} 768 \mathrm{I} \\
\text { EGFR Exon } 21 \mathrm{~L} 858 \mathrm{R}\end{array}$ & & \\
\hline 15 & EGFR Exon 21 L858R & & \\
\hline 33 & & KRAS Exon 2 G12C & EGFR Exon 13 R521K \\
\hline 38 & EGFR Exon 19 E746_A750del & & \\
\hline 39 & EGFR Exon 18 G721S & & \\
\hline 40 & & EGFR Exon 20 S784T & \\
\hline 41 & & EGFR Exon 19 P753P & \\
\hline 48 & EGFR Exon 19 T751_E758del & & \\
\hline 50 & EGFR Exon 19 L747_P753delinsS & & \\
\hline 53 & & $\begin{array}{l}\text { EGFR Exon } 20 \text { A767_V469 } \\
\text { duplication } \\
\text { ERBB2 amplification }\end{array}$ & \\
\hline 55 & EGFR Exon 19 E746_A750del & & \\
\hline 57 & EGFR Exon 19 E746_T751delinsA & & \\
\hline 63 & & $\begin{array}{l}\text { EGFR Exon } 20 \text { T790M } \\
\text { EGFR Exon } 20 \text { C797S }\end{array}$ & \\
\hline 69 & EGFR Exon 18 I706S & & \\
\hline 73 & & $\begin{array}{l}\text { EGFR Exon } 20 \text { H773fs } * 53 \text {, } \\
\text { Met amplification }\end{array}$ & \\
\hline
\end{tabular}




> continuation from the previous page
\begin{tabular}{|l|l|l|l|}
\hline 78 & & $\begin{array}{l}\text { EGFR Exon 20 K806I, } \\
\text { Met Exon 14 R988H, } \\
\text { Met Exon 14 Y989C }\end{array}$ & \\
\hline 80 & & KRAS Exon 2 G12C & EGFR Exon 13 R521K \\
\hline 81 & EGFR Exon 20 D807fs *90 & & \\
\hline 84 & EGFR Exon 20 V786L & & \\
\hline 85 & EGFR Exon 21 A864T & & \\
\hline 86 & EGFR Exon 19 L747_T751del & $\begin{array}{l}\text { KRAS Exon 2 G12S, } \\
\text { Met Exon 14 E1012K }\end{array}$ & \\
\hline 88 & $\begin{array}{l}\text { EGFR Exon 19 E746_A750delELRE A, } \\
\text { EGFR Amplification }\end{array}$ & & \\
\hline
\end{tabular}

Table 4. Detailed molecular findings of adenocarcinoma and non-small cell lung cancer-not otherwise specified patients other than EGFR mutations.

\begin{tabular}{|c|c|c|c|}
\hline$\#$ & Activating Mutation(s) & Resistance Mutation(s) & Polymorphism \\
\hline 5 & & $K R A S$ exon $2 \mathrm{G} 12 \mathrm{C}$ & \\
\hline 33 & & KRAS exon $2 \mathrm{G} 12 \mathrm{C}$ & $E G F R$ exon $13 \mathrm{R} 521 \mathrm{~K}$ \\
\hline 37 & & KRAS exon $2 \mathrm{G} 12 \mathrm{~V}$ & \\
\hline 49 & & $K R A S$ exon $2 \mathrm{G} 12 \mathrm{~V}(\mathrm{c} .35 \mathrm{G}>\mathrm{T})$ & \\
\hline 80 & & KRAS exon 2 G12C & EGFR exon $13 \mathrm{R} 521 \mathrm{~K}$ \\
\hline 86 & $\begin{array}{l}\text { EGFR exon } 19 \mathrm{~L} 747 \mathrm{~T} 751 \mathrm{del} ; \\
M E T \text { exon } 14 \mathrm{E} 1012 \overline{\mathrm{K}}\end{array}$ & $K R A S$ exon $2 \mathrm{G} 12 \mathrm{~S}$ & \\
\hline 87 & & $K R A S$ exon $2 \mathrm{G} 12 \mathrm{D}$ & \\
\hline 90 & & KRAS intron 2 & \\
\hline 14 & & MET amplification & \\
\hline 73 & & $\begin{array}{l}\text { EGFR exon } 20 \mathrm{H} 773 \mathrm{fs} * 53 ; \\
M E T \text { amplification }\end{array}$ & \\
\hline 78 & & \begin{tabular}{|l|}
$E G F R$ exon $20 \mathrm{~K} 806 \mathrm{I}$ \\
MET exon $14 \mathrm{R} 988 \mathrm{H} ;$ \\
MET exon $14 \mathrm{Y} 989 \mathrm{C}$ \\
\end{tabular} & \\
\hline 44 & $A L K$ exon $29 \mathrm{~K} 1491 \mathrm{R}$ & $E R B B 2$ exon $17 \mathrm{I} 655 \mathrm{~V}$ & EGFR exon $13 \mathrm{R} 521 \mathrm{~K}$ \\
\hline 95 & $A L K$ exon $22 \mathrm{C} 1156 \mathrm{~S}$ & & \\
\hline 31 & & ERBB2 amplification & \\
\hline 53 & & $\begin{array}{l}\text { EGFR exon 20 A767_V469dup; } \\
\text { ERBB2 amplification }\end{array}$ & \\
\hline 79 & & $E R B B 2$ exon $17 \mathrm{I} 655 \mathrm{~V}$ & \\
\hline 58 & & & $\begin{array}{l}\text { PDGFRA c. } 236 \mathrm{G}>\mathrm{A}(\mathrm{G} 79 \mathrm{D}) ; \\
\text { KIT c. } 2362-77 \mathrm{G}>\mathrm{A}\end{array}$ \\
\hline 18 & $B R A F$ exon $11 \mathrm{G} 469 \mathrm{E}$ & & \\
\hline
\end{tabular}

Table 5. Second liquid biopsy results in adenocarcinoma and non-small cell lung cancer-not otherwise specified patients.

\begin{tabular}{|c|c|c|c|}
\hline$\#$ & First Liquid Biopsy & Second Liquid Biopsy & Prior Treatment \\
\hline 2 & \begin{tabular}{|l|}
$E G F R$ exon 19 A750P; \\
$E G F R$ exon 19 L747 E749del \\
\end{tabular} & no variant detected & erlotinib \\
\hline 10 & EGFR exon $18 \mathrm{G} 721 \overline{\mathrm{V}}$ & no variant detected & paclitaxel + carboplatin \\
\hline 13 & no variant detected & no variant detected & paclitaxel + carboplatin \\
\hline 15 & EGFR exon $21 \mathrm{~L} 858 \mathrm{R}$ & $B R A F$ exon 15 R603* & erlotinib \\
\hline 31 & ERBB2 amplification & no variant detected & paclitaxel + carboplatin \\
\hline 38 & EGFR exon 19 E746_A750del & no variant detected & erlotinib \\
\hline 55 & EGFR exon 19 E746_A750del & $\begin{array}{l}E G F R \text { exon } 19 \text { E746_A750del; } \\
\text { EGFR exon } 20 \mathrm{~T} 790 \mathrm{M}\end{array}$ & erlotinib \\
\hline 95 & $A L K$ exon $22 \mathrm{C} 1156 \mathrm{~S}$ & no variant detected & crizotinib \\
\hline
\end{tabular}




\section{DISCUSSION}

The treatment of NSCLC has become more successful and individualized using targeted protocols according to molecular subtypes. The earlier attempts with targeted treatments involved unselected patients and did not result in as good outcomes as anticipated. Regarding treatment with the epidermal growth factor receptor tyrosine kinase inhibitors (EGFR-TKIs) in unselected patients, while erlotinib increased median survival by 2 months in previously treated NSCLC patients [9], gefitinib did not improve survival in a similar population [10].

However, targeted treatment in patients selected according to molecular analyses resulted in unprecedented outcomes. For instance, a prospective study in patients with EGFR mutant LC, the response rate to targeted treatment was over $60.0 \%$ [11]. Crizotinib resulted in a similar success rate in LC patients with $A L K$ rearrangements [12]. First line treatment with dabrafenib/trametinib in NSCLC patients carrying $B R A F \mathrm{~V} 600 \mathrm{E}$ mutations (seen in $2.0 \%$ of patients with NSCLC) resulted in a response rate of $64.0 \%$ and 10 months of progression-free survival [13]. The identification of the even rarer variants such as $M E T$ gene amplification and $E R B B 2$ mutations led to the discovery of new treatment pathways. In 2014, the NCCN recommended utilization of wider panels including $B R A F, E R B B 2$ (HER2), MET, RET and ROS1 in addition to EGFR and $A L K$. The reason for their recommendation to widen the mutation panel was the ever-increasing success with novel treatments targeted to oncogenic driver mutations in comparison to cytotoxic and immune checkpoint inhibitor therapies.

These tests were performed step by step, therefore large tissue samples were inevitably needed [14]. Various clinical studies indicate that attainment of ccfDNA is an appropriate means of detecting mutations $[15,16]$. Recently, the advantages of plasma-based liquid biopsy such as accessibility, practical use and reproducibility, rendered it a good choice in patients with NSCLC. Tissue biopsies usually bear the difficulty of access and may provide insufficient samples for genetic testing due to the tumor heterogeneity. Furthermore, the need for a repeat biopsy after lack of response to treatment may be a cause of significant morbidity. The ASSESS study reported a high degree of concordance between plasma-based ccfDNA and tissue or cytology samples in NSCLC [17].

We aimed to report our NGS results using liquid biopsy samples in patients with metastatic NSCLC. The most common mutation was regarding the EGFR gene. Some of the patients had concomitant driver and resistance mutations. The second most commonly detected gene was $K R A S$. While the frequency of EGFR mutation in AC in the western populations was $19.2 \%$; it was seen in $47.9 \%$ of Asian patients. The rates of KRAS mutations was $26.1 \%$ in the western and $11.2 \%$ in Asian populations [18]. Turkey lies between the western populations and Asia, and the frequencies of $E G F R$ and KRAS mutations in the present study were $31.4 \%$ (17.4\% activating) and $9.3 \%$, respectively. The other targets of individualized treatment protocols include EML4-ALK fusion and variants of MET, ERBB2, BRAF, $P D G F R A$ and KIT. The most common variants detected in patients with $\mathrm{AC}$ were in $E G F R$ and $K R A S$ genes. The two most commonly detected variants were also seen in patients with SCC in a diverse frequency according to their ethnic origin [19]. In the present study, EGFR and MET variants were also observed in patients with SCC. The activation of the MET signaling pathway is suggested to cause resistance to EGFR-TKI treatment. Crizotinib may provide successful outcomes in patients with MET overexpression [20]. Therefore, target mutations should be sought using liquid biopsy in patients with SCC. Despite the dramatic response to molecular targeted treatments, nearly every medication is associated with development of resistance. It is also important to understand the molecular pathways of NSCLC in order to understand mechanisms of resistance to medications [21].The understanding of resistance mechanisms against targeted treatments led to the development of second line treatment options specific to EGFR and $A L K$ mutations. Moreover, the growing need for repeat biopsies in patients with LC is associated with a substantial morbidity risk. Furthermore, the paucity of centers for transthoracic biopsies in many healthcare systems cause a logistics problem. Therefore, the opportunity of following up patients with LC using blood-based tests is an important topic of interest in the technologically developing world $[22,23]$. While the most common mechanism of resistance against first and second line EGFR-TKIs is the EGFR T790M mutation, $M E T$ and $E R B B 2$ amplification is responsible for a smaller proportion of resistance [24]. In the present study, we also aimed to investigate the causes of resistance in the eight patients with progressive disease after the first treatment, and found the following causes: new onset EGFR T790M mutation, erased target mutation and a new onset $B R A F$ gene variant. The present study has some strong points as well as limitations. New onset variants that are potential targets of alternative treatment options and the disappearance of the previous mutations showed the necessity of repeated liquid biopsy studies. Liquid biopsy has a strong advantage in NSCLC because of the difficulties of repeated interventional procedures and accessibility problems. To the best of our knowledge, this study is the first to report on the real-world use of a comprehensive ccfDNA with clinical follow-ups and NGS platforms in the molecular diagnosis of patients with NSCLC in a Turkish population. The main limitation of working with the circulating tumor cell as a liquid biopsy 
source is the fact that the sample obtained in patients with early stage cancer may not contain sufficient amounts of tumor-derived material and sometimes information from tumor tissue may be difficult to separate information from intact cells [25]. An example of this is PDGFRA c.236G $>\mathrm{A}$ (G79D), KIT c.2362-77G $>$ A variants, which are not compatible with the diagnosis and clinical feature of a patient and was evaluated as a polymorphism. The second liquid biopsy sampling was performed only in a small number of cases and this was the other limitation of this study.

\section{CONCLUSIONS}

In the present study we reported the results of targeted multigene panel NGS analysis of ccfDNA in Turkish patients with NSCLC. We demonstrated the feasibility of this advanced diagnostic method and identified mutations of resistance and potential treatment target. We suggest that the non invasive liquid biopsy will have a great clinical importance in the management of patients with NSCLC when obtained tissue samples are inadequate or repeat biopsy is difficult.

\section{ACKNOWLEDGMENTS}

Part of this study was presented as a verbal presentation at the National Lung Cancer Congress on October 4-7, 2018 at Bodrum, Turkey. Author contributions: M. Buyuksimsek conceived the study and all authors designed the study. M. Buyuksimsek, M. Togun, B. Sahin, A. Evren Yetisir, A. Ogul and M. Tohumcuoglu collected the data. I. Boga performed the NGS studies. I. Boga and A. Bisgin analyzed the data. M. Buyuksimsek, I. Oguz Kara, M. Togun, M. Tohumcuoglu, B. Sahin and A. Bisgin interpreted the results. M. Buyuksimsek, I. Oguz Kara, H. Erdem Sumbul and A. Bisgin drafted the manuscript with critical revisions from B. Sahin, M. Togun and M. Tohumcuoglu. All authors approved the final version.

Declaration of Interest. The authors report no conflicts of interest. The authors alone are responsible for the content and writing of this article.

\section{REFERENCES}

1. Travis WD, Brambilla E, Burke AP, Marx A, Nicholson AG. Pathology and Genetics of Tumors of the Lung, Pleura, Thymus, and Heart, 4th ed. Lyon, France: International Agency for Research on Cancer Press; 2015.

2. Lindeman NI, Cagle PT, Aisner DL, Arcila ME, Beasley MB, Bernicker EH, et al. Updated molecular testing guideline for the selection of lung cancer patients for treatment with targeted tyrosine kinase inhibitors: Guideline from the college of American Pathologists, the International Association for the Study of Lung Cancer, and the Association for Molecular Pathology. Arch Pathol Lab Med. 2018; 142(3): 321-346.

3. Newman AM, Bratman SV, To J, Wynne JF, Eclov NC, Modlin LA, et al. An ultrasensitive method for quantitating circulating tumor DNA with broad patient coverage. Nat Med. 2014; 20(5): 548-554.

4. Thompson JC, Yee SS, Troxel AB, Savitch SL, Fan R, Balli D, et al. Detection of therapeutically targetable driver and resistance mutations in lung cancer patients by next-generation sequencing of cell-free circulating tumor DNA. Clin Cancer Res. 2016; 22(23): 5772-5782.

5. Schwaederlé MC, Patel SP, Husain H, Ikeda M, Lanman RB, Banks KC, et al. Utility of genomic assessment of blood-derived circulating tumor DNA (ctDNA) in patients with advanced lung adenocarcinoma. Clin Cancer Res. 2017; 23(17): 5101-5111.

6. Sawada K, Kotani D, Bando H. The clinical landscape of circulating tumor DNA in gastrointerstinal malignancies. 2018; 8: 263. doi: 10.3389/fonc.2018.00263. eCol lection 2018.

7. Esposito AR, Pasquale R, Fenizia F, Rachiglio AM, Roma C, Bergantino F, et al. The role of circulating free DNA in the management of NSCLC. Expert Rev Anticancer Ther. 2019; 19(1): 19-28.

8. Uchida J, Kato K, Kukita Y, Kumagai T, Nishino K, Daga $\mathrm{H}$, et al. Diagnostic accuracy of non invasive genotyping of EGFR in lung cancer patients by deep sequencing of plasma cell-free DNA. Clin Chem. 2015; 61(9): 1191-1196.

9. Shepherd FA, Rodrigues Pereira J, Ciuleanu T, Tan EH, Hirsh V, Thongprasert S, et al. Erlotinib in previously treated non-small-celllung cancer. N Engl J Med. 2005; 353(2): 123-132.

10. Thatcher N, Chang A, Parikh P, Rodrigues Pereira J, Ciuleanu T, von Pawel J, et al. Gefitinib plus best supportive care in previously treated patients with refractory advanced non-small-cell lung cancer: Results from a randomized, placebo controlled, multicentre study (Iressa Survival Evaluation in Lung Cancer). Lancet. 2004; 366(9496): 1527-1537.

11. Rosell R, Carcereny E, Gervais R, Vergnenegre A, Massuti B, Felip E, et al. Erlotinib versus standard chemotherapy as first line treatment for European patients with advanced EGFR mutation-positive nonsmall-cell lung cancer (EURTAC): A multicentre, open-label, randomised phase 3 trial. Lancet Oncol. 2012; 13(3): 239-246. 
12. Kwak EL, Bang YJ, Camidge DR, Shaw AT, Solomon B, Maki RG, et al. Anaplastic lymphoma kinase inhibition in nonsmall-cell lung cancer. N Engl J Med. 2010; 363(18): 1693-1703.

13. Planchard D, Smit EF, Groen HJM, Mazieres J, Besse $\mathrm{B}$, Helland $\AA$, et al. Dabrafenib plus trametinib in patients with previously untreated BRAFV600E-mutant metastatic non-small-cell lung cancer: An open-label, phase 2 trial. Lancet Oncol. 2017; 18(10): 1307-1316.

14. Ettinger DS, Wood DE, Akerley W, Bazhenova LA, Borghaei H, Camidge DR, et al. Non-small cell lung cancer, version 6.2015. J Natl Compr Canc Netw. 2015; 13(5): 515-524.

15. Mok T, Wu YL, Lee JS, Yu CJ, Sriuranpong V, Sandoval-Tan $\mathrm{J}$, et al. Detection and dynamic changes of EGFR mutations from circulating tumor DNA as a predictor of survival outcomes in NSCLC patients treated with first-line intercalated erlotinib. Clin Cancer Res. 2015; 21(14): 3196-3203.

16. Douillard JY, Ostoros G, Cobo M, Ciuleanu T, Cole $\mathrm{R}$, McWalter G, et al. Gefitinib treatment in EGFR mutated caucasian NSCLC: Circulating-free tumor DNA as a surrogate for determination of EGFR status. J Thorac Oncol. 2014; 9(9): 1345-1353.

17. Reck M, Hagiwara K, Han B, Tjulandin S, Grohé $\mathrm{C}$, Yokoi T, et al. ctDNA determination of EGFR mutation status in European and Japanese patients with advanced NSCLC: The ASSESS study. J Thorac Oncol. 2016; 11(10): 1682-1689.

18. Dearden S, Stevens J, Wu YL, Blowers D. Mutation incidence and coincidence in non small-cell lung cancer: Meta-analyses by ethnicity and histology (mutMap). Ann Oncol. 2013; 24(9): 2371-2376.
19. Zhang X-C, Wang J, Shao G-G, Wang Q, Qu X, Wang $\mathrm{B}$, et al. Comprehensive genomic and immunological characterization of Chinese non-small cell lung cancer patients. Nat Commun. 2019; 10(1): 1772.

20. Song Z, Wang H, Yu Z, Lu P, Xu C, Chen G, et al. De novo MET amplification in Chinese patients with non-small-cell lung cancer and treatment efficacy with crizotinib: A multicenter retrospective study. Clin Lung Cancer. 2018; 20(2)): e171-e176.

21. Asao T, Takahashi F, Takahashi K. Resistance to molecularly targeted therapy in non-small-cell lung cancer. Respir Investig. 2019; 57(1): 20-26. doi: 10.1016/j.resinv. 2018.09.001. Epub 2018 Oct 4.

22. Konduri K, Gallant JN, Chae YK, Giles FJ, Gitlitz BJ, Gowen $\mathrm{K}$, et al. EGFR fusions as novel therapeutic targets in lung cancer. Cancer Discov. 2016; 6(6): 601-611.

23. Mok TS, Wu YL, Ahn MJ, Garassino MC, Kim HR, Ramalingam SS, et al. Osimertinib or platinumpemetrexed in EGFR T790M-positive lung cancer. N Engl J Med. 2017; 376(7): 629-640.

24. Rolfo C, Giovannetti E, Hong DS, Bivona T, Raez LE, Bronte G, et al. Novel therapeutic strategies for patients with NSCLC that do not respond to treatment with EGFR inhibitors. Cancer Treatment Rev. 2014; 40(8): 990-1004.

25. Linda K, Mikael J, Kjell G, Jonas N. Liquid biopsies in lung cancer - time to implement research technologies in routine care? Ann Transl Med. 2017; 5(13): 278 . 
